\title{
Lehren aus dem regionalen Emissionshandel in Nordamerika
}

\author{
Um die immensen Herausforderungen zu be- \\ wältigen, muss das Pariser Klimaabkommen \\ auf allen Regulierungsebenen konkretisiert wer- \\ den. Lokale und regionale Emissionshandels- \\ systeme bieten gerade in Situationen nationalen \\ Politikversagens Chancen für den Klimaschutz \\ von unten. Von Sven Rudolph, Toru Morotomi, \\ Takeshi Kawakatsu und Achim Lerch
}

\section{Der theoretische Hintergrund}

Die neoklassische Umweltökonomie hat überzeugend zeigen können, dass der Emissionshandel, eine Vielfalt an Vorzügen besitzt (Endres 2011). Das Instrument implementiert eine absolute Emissionshöchstmenge für einen geografischen Raum (Cap), verteilt Emissionsrechte an die Emittenten (Distribute) mit der Vorgabe, nur so viele Emissionen ausstoßen zu dürfen, wie auch Emissionsrechte gehalten werden, überlässt die Mikroallokation aber einem privaten Markt, auf dem Emittenten und andere Interessierte Emissionsrechte kaufen und verkaufen dürfen (Trade).

Damit folgt der Emissionshandel der Idee von Daly (1996), nach der in einer Postwachstumsgesellschaft die zentralen Entscheidungen über Ausmaß (Scale), Verteilung (Distribution) und Allokation separiert, von unterschiedlichen Entscheidungsträgern und in der genannten Prioritätenfolge getroffen werden müssen. So lassen sich nachhaltige Emissionshandelssysteme gestalten, die sowohl ökonomischen und ökologischen als auch Ansprüchen der sozialen Gerechtigkeit genügen ( $\mathrm{Ru}$ dolph et al. 2012).

Der Emissionshandel erreicht bei theoriekonformer Ausgestaltung ein gegebenes umweltpolitisches Ziel, konkret eine staatlich festgelegte Emissionsobergrenze, zielgenau. Dies geschieht zu volkswirtschaftlich minimalen Kosten. Zusätzlich werden kontinuierlich Innovationsanreize zur Verbesserung der Vermeidungstechnologie gesetzt. Zwar sind die Effizienzgewinne am größten, wenn der Anwendungsbereich maximal ist, sodass im Klimaschutz ein globaler Markt für Treibhausgase die erstbeste Lösung wäre. Angesichts der technischen und politischen Schwierigkeiten einer solchen globalen Lösung, stellt eine Verknüpfung (Linking) nationaler, regionaler oder lokaler Märkte allerdings die zweitbeste und politisch realistischere Lösung dar (Ranson/Stavins 2015). Jüngste Studien zeigen, dass sogar eine nachhaltige Verknüpfung möglich ist (Lenz et al. 2014, Rudolph et al. 2016).

In der umweltpolitischen Praxis sind seit den ersten Emissionshandelssystemen in der U.S.-amerikanischen Luftreinhaltepolitik der 1970er und 80er Jahre vielfältige, teils ambivalente Erfahrungen gesammelt worden (Schmalensee/Stavins 2015). In der Klimapolitik sind die Erfahrungen ebenfalls zwiespältig. Nach der Rahmensetzung durch die flexiblen Mechanismen des Kyoto-Protokolls etablierte Europa 2005 das bis heute größte klimapolitische Emissionshandelssystem. Vor allem das Politikversagen bei der Setzung des Cap und die globale Finanzkrise 2008 führten zu einem Preissturz von anfangs fast 30 Euro pro Tonne auf nahe null im Jahr 2007 und jüngst rund 5,50 Euro im November 2016. Wenngleich niedrige Preise weder die statische Kosteneffizienz noch die exakte Zielerreichung gefährden, so wird vor allem befürchtet, dass die Innovationsanreize für die notwendige Dekarbonisierung der europäischen Wirtschaft nicht ausreichen (Kemfert 2015).

Weitere klimapolitische Emissionshandelssysteme wurden auf nationaler Ebene in der Schweiz, Neuseeland, Kasachstan und Südkorea implementiert, während sie in anderen Ländern wie Australien und Japan zumindest zeitweise wirksam waren. Die größten aktuellen Hoffnungen bestehen hinsichtlich eines nationalen Emissionshandels in China. Die stärkste Dynamik hat sich jüngst aber auf subnationaler Ebene entfaltet mit lokalen Systemen in Tokyo und in verschiedenen chinesischen Städten wie Peking und Shanghai, aber auch auf regionaler Ebene in Kanada und den USA (ICAP 2016).

Dieser Trend einer marktbasierten Klimapolitik von unten wird nicht nur von klimawissenschaftlichen Fakten wie der zunehmenden Relevanz von Treibhausgas-Emissionen aus (Groß-)Städten untermauert. (OECD 2009). Vielmehr findet sie auch starke Fürsprecher in Forschung und Politik, wie beispielsweise jüngste Vorschläge für subglobale Klimapolitikallianzen (WBGU 2010), für einen Club of Carbon Markets (Keohane et al. 2015) oder für eine Subnational Global Climate Leadership (Under2 2016) zeigen.

Diese Bewegung greift letztlich Ostroms (2009) Ansatz einer polyzentrischen Klimapolitik auf und wird auch vom neuen umweltpolitischen Föderalismus unterstützt (Morotomi 2014, Oates 2004). Nachdem Charles Tiebout bereits 1956 bei der Bereitstellung öffentlicher Güter den Wettbewerb zwischen Kommunen über ein „voting by feet“ positiv bewertet hattet, warnte vor allem Richard Stewart später vor einem „race to the bottom“ und plädierte für eine Zentralisierung gerade bei grenzüberschreitenden Externalitäten. Jüngst wird aber betont, dass 
Regionen und Kommunen selbst bei globalen Umweltproblemen eine wichtige Rolle spielen. Sie könnten, so die zentralen Argumente, als umweltpolitische Versuchslabore dienen, lokale sozio-ökonomische und technische Gegebenheiten wie beispielsweise die Emissionsstruktur besser berücksichtigen und eher den Präferenzen der jeweils ansässigen Bevölkerung Rechnung tragen. So würden letztendlich sogar Effizienzgewinne realisiert werden, wenn beispielsweise Großstädte wie Tokyo mit einem lokalen $\mathrm{CO}_{2}$-Emissionshandel anspruchsvolle Klimaschutzziele über kosteneffiziente Emissionsreduktionen im Gebäudebereich erreichen, um so den vergleichsweise proaktiven Präferenzen der lokalen Bevölkerung gerade mit Blick auf die Olympischen Spiele 2020 besser zu entsprechen (Rudolph/Morotomi 2015). Verschiedene U.S.-amerikanische Bundesstaaten und kanadische Provinzen vorwiegend an der relativ umweltorientierten Ost- und Westküste haben sich diese Idee ebenfalls zu eigen gemacht.

\section{Regionaler Treibhausgas-Emissionshandel in Nordamerika}

\subsection{Regional Greenhouse Gas Initiative (RGGI)}

Mit RGGI wurde im Jahr 2009 das erste Treibhausgas-Emissionshandelssystem in den USA etabliert und 2014 grundlegend reformiert (RGGI 2013). RGGI setzt sich aus juristisch unabhängigen, aber über eine Rahmenordnung (Model Rule) in zentralen Ausgestaltungsmerkmalen vereinheitlichten Systemen in aktuell neun Bundesstaaten des U.S.-amerikanischen Nordostens zusammen. RGGI begrenzt die Gesamtemissionen von $\mathrm{CO}_{2}$ aus 163 großen stromerzeugenden Anlagen auf fossiler Basis, deckt damit allerdings nur 20\% der Treibhausgas-Emissionen der teilnehmenden Bundesstaaten ab. Das ursprünglich großzügige Cap von 188 Millionen Tonnen $\mathrm{CO}_{2}$ wurde im Jahr 2014 aufgrund einer erheblichen Überalloka- tion um mehr als 50\% auf 91 Millionen Tonnen reduziert und verkleinert sich seit 2015 jedes Jahr um weitere 2,5\%. Für 2020 wird so eine Gesamtemissionsreduktion von etwa $50 \%$ gegenüber 1990 erwartet.

Die Model Rule verlangt von den teilnehmenden Bundesstaaten einen Versteigerungsanteil von mindestens 25\% bei der Erstvergabe der $\mathrm{CO}_{2}$-Rechte. Tatsächlich beträgt dieser aber im Durchschnitt mehr als 90\%. Emissionsrechte können auf vierteljährlich stattfindenden Auktionen oder auf Sekundärmärkten erworben werden. Außerdem müssen mindestens 25\% der Einnahmen für konsumentenseitige Förderprogramme im Bereich Energieeffizienz und erneuerbare Energien verwendet werden; aber auch direkte Unterstützungen für einkommensschwache Haushalte sind möglich. Der tatsächliche Versteigerungsanteil von über $90 \%$ erlaubt allerdings auch anderweitige Verwendungen. So werden im Durchschnitt rund zwei Drittel der Einnahmen in den Klimaschutz investiert und jeweils 15\% gehen in die Unterstützung einkommensschwacher Haushalte und die Haushaltskonsolidierung.

Zusätzliche Flexibilität bei der Erfüllung der gesetzlichen Vorgaben durch die Emittenten bieten das sogenannte Banking, also die Möglichkeit des Aufbewahrens von Emissionsrechten sowie die quantitativ und qualitativ begrenzte Nutzung von Offsets, Emissionsreduktionskrediten, die außerhalb des eigentlichen RGGI-Emissionshandelssystems generiert werden.

Zur Preiskontrolle nutzt RGGI einen Preiskorridor (Price Collar), bei dem als untere Grenze ein Mindestpreis von anfangs 1,89 US\$ mit einem jährlichen Anstieg von 2,5\% plus Inflationsausgleich gesetzt wurde. Die Obergrenze wird über eine Kostenbeschränkungsreserve (Cost Containment Reserve) bestimmt, aus der rund 10 Millionen Emissionsrechte erworben werden können, wenn der $\mathrm{CO}_{2}$-Preis 4 US \$ in 2014, 6 US \$ in 2015, 8 US \$ in 2016 oder 10 US \$ in 2017 übersteigt. Ab 2018 steigt der Preis jährlich um 2,5\% plus Inflationsausgleich.

\section{Wie sich Zukunft gestalten lässt}

Wie lässt sich eine "Welt des Weniger" gestalten? Harald Welzer und Bernd Sommer präsentieren praxiserprobte Konzepte - von der Gemeinwohlökonomie bis zu den Transition Towns.
Bernd Sommer, Harald Welzer

\section{Transformationsdesign Wege in eine zukunftsfähige Moderne}

oekom verlag, München

240 Seiten, Broschur

12,95 Euro

ISBN: 978-3-86581-845-4

Im Handel ab dem: 20.02.2017

Auch als E-Book erhältlich

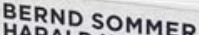

HARALD SOMMER

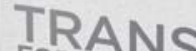

FORMATS

DESIGN

Wege in eine

Moderne

nitovion

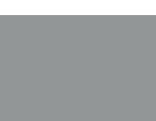

oekom.de

DIE GUTEN SEITEN DER ZUKUNFT 
Am Ende einer jeweils dreijährigen Kontrollperiode müssen 100\% der Emissionen durch Emissionsrechte abgedeckt werden. Eine Nichterfüllung der Vorgaben führt zu erheblichen Sanktionen mit Strafzahlungen von bis zu 25.000 US\$ und einer Nacherfüllungspflicht im Verhältnis $3 \mathrm{zu} 1$.

Die bisherigen Erfahrungen mit RGGI sind ambivalent (Potomac Economics 2016, RGG I 2016). Nach der Revision von 2014 folgt die Ausgestaltung von RGGI in wichtigen Teilen wie der bindenden Emissionsgesamtmenge, der kostenpflichtigen Erstvergabe und der ökologisch-sozial ausgerichteten Aufkommensverwendung zwar durchaus anspruchsvollen Nachhaltigkeitskriterien, widerspricht diesen aber auch hinsichtlich des engen Anwendungsbereichs. Aus umweltökonomischer Sicht lassen sich die 100\% Erfüllung der gesetzlichen Vorgaben durch die Emittenten und eine Unterschreitung des Cap um 4,9\% in 2013, ein aktiver und kompetitiver Markt für Emissionsrechte und ein nach der Verknappung der Emissionsrechte steigender $\mathrm{CO}_{2}$-Preis positiv hervorheben. Auch für RGGI gilt allerdings, dass ein $\mathrm{CO}_{2}$-Preis von rund 7,50 US\$ Ende 2015 nicht ausreicht, um die für eine Dekarbonisierung notwendigen Innovationsanreize zu setzen.

\subsection{Western Climate Initiative (WCI)}

Die Western Climate Initiative (WCI) strebt seit 2008 die Etablierung subnationaler Treibhausgas-Märkte an, die dann über ein Linking verknüpft werden sollen. Kalifornien und Québec haben solche regionalen Märkte 2013 implementiert und 2014 verknüpft, während Ontario den Start für 2017 plant. Manitoba und Washington State denken ebenfalls über die Einführung eigener Systeme nach. Die drei ausgearbeiteten Programme orientieren sich an der WCI-Model-Rule (WCI 2008) und unterscheiden sich daher nur in Einzelaspekten (CARB 2013, GoO 2016, GdQ 2014).

Die Teilnahme für die einbezogenen Emittenten ist in allen drei Systemen verpflichtend und die Programme decken jeweils die Emissionen aller Treibhausgase des Kyoto-Protokolls aus großen stationären Quellen (Industrie, Stromerzeugung inklusive Stromimporte) ab; seit 2015 sind in Kalifornien und Québec und ab 2017 in Ontario auch Emissionen aus der Nutzung flüssiger und gasförmiger fossiler Brennstoffe in kleineren Anlagen, in Gebäuden und im Transportsektor inkludiert (Upstream), die über die Vertreiber der Energieträger abgerechnet werden. In Kalifornien betrifft dies rund 450 Einrichtungen, in Québec 80 und in Ontario 150. Die Gesamtabdeckung liegt damit in den Regionen bei rund 85\% der gesamten Treibhausgas-Emissionen.

Das Cap wurde in Kalifornien im Jahr 2013 auf 163 Millionen Tonnen begrenzt, mit der Ausweitung 2015 dann aber auf 395 Millionen Tonnen erhöht. Ein Reduktionspfad von 2-3\% jährlich wird für eine Emissionsreduktion von insgesamt rund 15\% im Jahr 2020 gegenüber 1990 sorgen. Québec startete mit einem Cap von 23 Millionen Tonnen, das 2015 auf 65 Millionen Tonnen erhöht wurde. Mit einer jährlichen Reduktion von 1-2\% wird 2020 eine Emissionsreduktion von 25\% unter dem
1990er-Niveau erreicht. In Ontario werden zunächst 142 Millionen Emissionsrechte ausgegeben. Das Cap nimmt dann um rund 4,4\% pro Jahr ab, sodass im Jahr 2020 eine Emissionsreduktion von rund 15\% unter dem Niveau von 1990 erreicht wird.

In Kalifornien erhalten Stromunternehmen und Industrieanlagen rund 90\% der Emissionsrechte kostenfrei, wobei die Stromerzeuger den ökonomischen Wert dieser Rechte allerdings über konsumentenseitige Förderprogramme an die Stromverbraucher zurückgeben müssen. Für Industrieanlagen soll der Versteigerungsanteil kontinuierlich angehoben werden. Die Vertreiber fossiler Energieträger hingegen müssen alle benötigten Emissionsrechte käuflich erwerben. In Québec und Ontario müssen grundsätzlich 100\% der Emissionsrechte käuflich erworben werden, wobei für Industrieanlagen im internationalen Wettbewerb ebenfalls Ausnahmen gelten: Sie erhalten $80-100 \%$ der Emissionsrechte kostenfrei, wobei auch hier der Anteil kostenloser Zuteilung abgeschmolzen werden soll. Emissionsrechte können auf vierteljährlich stattfindenden Auktionen oder auf Sekundärmärkten erworben werden.

Die Versteigerungserlöse müssen in Kalifornien zu mindestens $85 \%$ an die Stromverbraucher über konkrete Emissionsreduktionsmaßnahmen im Bereich erneuerbarer Energien oder Energieeffizienz zurückgegeben werden. Außerdem müssen 25\% der Investitionen zugunsten von einkommensschwachen Kommunen erfolgen. In Québec und Ontario werden die gesamten Auktionserlöse in Klimaschutzprojekte investiert. Teile des Aufkommens können aber auch dort zur Abmilderung negativer Verteilungswirkungen genutzt werden.

Zur Flexibilisierung ist in allen drei Regionen Banking und qualitativ und quantitativ begrenzt die Nutzung von Offsets erlaubt.

Zur Kostenkontrolle existiert ein abgestimmter Preiskorridor: Aus einer Kostenbeschränkungsreserve (Cost Containment Reserve), die mit einer ansteigenden Zahl an Emissionsrechten aus dem Cap ausgestattet ist (1\% in 2013-14, 4\% in 2015-17, 7\% in 2018-20), können Emissionsrechte in drei gleich großen Segmenten zu Preisen von 40, 45 und 50 US\$ erworben werden. Als Preisuntergrenze wurde bei den Auktionen ein Mindestpreis von 10 US\$ gesetzt. Beide Grenzen werden jährlich um 5\% plus Inflationsausgleich angehoben.

Am Ende einer jeden dreijährigen Erfüllungsperiode müssen 100\% der Emissionen mit entsprechenden Emissionsrechten abgedeckt werden. Die Nichterfüllung wird mit empfindlichen Geldbußen und einer Nacherfüllungspflicht von Verhältnis eins zu drei bzw. eins zu vier in Kalifornien geahndet.

In der Bewertung gilt auch für die WCI eine teilweise Erfüllung anspruchsvoller Nachhaltigkeitskriterien. Allerdings fallen die Reduktionsziele hier eher schwach aus und bei der Erstvergabe wird ein signifikanter Anteil der Emissionsrechte weiterhin kostenfrei zugeteilt, positiv ist allerdings die umfassende Abdeckung. Im Ergebnis sind aus umweltökonomischer Perspektive eine 99\%-Erfüllung der Vorgaben durch die Emittenten, eine Emissionsgesamtmenge, die sogar knapp unter- 
halb des Cap von 2014 liegt, und ein aktiver und kompetitiver Markt für Emissionsrechte hervorzuheben (EDF 2015). Gleichwohl bewegt sich der Emissionsrechtepreis weiterhin gerade noch über dem Minimumpreis, was ein Fehlen von Knappheit andeutet.

\section{Model Rules und Carbon Pricing Benchmarks in Nordamerika}

Sowohl bei RGGI als auch bei der WCI haben überregionale Model Rules wesentlich zum Erfolg der Programme beigetragen. Sieben frühe RGGI-Teilnehmer leiteten ihre Model Rule im Jahr 2006 von einem vorausgegangenen Memorandum of Understanding (MOU) von $2005 \mathrm{ab}$ und orientierten sich dann, gemeinsam mit neuen Partnern, beim Aufbau prinzipiell unabhängiger Einzelstaatenprogramme an dieser. RGGI wurde dabei explizit etabliert, um Vorbild für ein nationales Emissionshandelssystem in den USA zu sein (Rudolph/Lerch 2012). Dabei wurde vor allem die auch für den EU-Emissionshandel mitverantwortliche klimapolitische Dynamik im ersten Jahrzehnt des neuen Jahrtausends genutzt. Auf nationaler Ebene scheiterte 2010 die Waxman-Markey-Bill mit einem Emissionshandelssystem als Kern aber trotzdem an parteipolitischen Kalkülen (Pooley 2010).

Dies führte in der WCI, die erst 2008 eine Model Rule vorgelegt hatte, zum Ausscheiden vieler Partner und generell zur Verzögerung der Umsetzung. Kalifornien und Québec starteten dann 2013 ihre eigenen Systeme auf der Basis der WCI Model Rule, verknüpften sie 2014 und erwarten nun zusätzliche Partner wie Ontario.

Auf der nationalen Ebene reagierte die Obama-Regierung auf das Scheitern der Waxman-Markey-Bill mit einer Strategie, die es nach einigen juristischen Umwegen, die wohl erst im Jahr 2017 endgültig abgeschlossen sein werden, der obersten Umweltbehörde Environmental Protection Agency (EPA) erlaubte, anspruchsvolle $\mathrm{CO}_{2}$-Emissionsstandards für Kohlekraftwerke zu erlassen (EPA 2015). Die konkrete Umsetzung des Clean Power Plan (CPP) obliegt im föderalen U.S.-System jedoch den Bundesstaaten. Der CPP enthält auch eine Model Rule für bundesstaatliche Emissionshandelssysteme, die bei Umsetzung eine Erfüllung der EPA-Standards nahezu garantieren. Die CPP Model Rule erlaubt zudem ausdrücklich die Kooperation zwischen Bundesstaaten entweder im Rahmen einer Glockenlösung (Bubble Policy), beispielsweise über ein gemeinsames Cap, oder über eine Verknüpfung (Linking) bereits etablierter Emissionshandelssysteme. Die CPP Model Rule könnte so einen bundesstaatlichen Wettbewerb um die beste Lösung anreizen und die Entwicklung eines supra-regionalen, vielleicht sogar nationalen Emissionshandelssystems in den USA von unten befördern.

In der kanadischen Provinz British Columbia war bereits 2008 statt eines Emissionshandels eine anspruchsvolle aufkommensneutrale Treibhausgassteuer eingeführt worden (Pedersen/Elgie 2015, Rudolph et al. 2014). Besteuert werden alle
Emissionen aus Verbrennungsprozessen mit nur wenigen Ausnahmen, sodass eine Abdeckung von 70\% erreicht wird. Der Steuersatz wurde von $10 \mathrm{C} \$$ pro Tonne $\mathrm{CO}_{2} \mathrm{e}$ in 2008 jährlich um 5 C\$ auf 30 C\$ im Jahr 2012 erhöht und dort eingefroren. Das Steueraufkommen wir vollumfänglich über Steuersenkungen bei den Einkommens- und Unternehmenssteuern rückverteilt. Zusätzlich können Steuererleichterungen für einkommensschwache Haushalte beantragt werden. Die bisherigen Erfahrungen sind weitgehend positiv: Bei solidem und mit Kanada vergleichbarem wirtschaftlichen Wachstum sind die Emissionen aus besteuerten Quellen zwischen 2008 und 2012 um 10\% gesunken, während sie im Rest von Kanada nur um rund $1 \%$ gesunken sind.

Angeregt vor allem von British Columbia und gestützt vom starkem politischen Zuspruch in der Bevölkerung kündigte der kanadische Premierminister Trudeau Anfang Oktober 2016 einen Carbon Pricing Benchmark an (Trudeau 2016). Demnach solle in allen kanadischen Provinzen ab 2018 ein Mindestpreis von $10 \mathrm{C} \$$ pro Tonne $\mathrm{CO}_{2}$-Äquivalent gelten, der dann um $10 \mathrm{C} \$$ pro Jahr ansteigt, um 202250 C\$ zu erreichen. Würden die Provinzen nicht agieren, so werde die kanadische Regierung eine solche Preistreppe implementieren, wobei die konkrete Instrumentierung bisher offengeblieben ist. Während British Columbia und Québec den Startwert bereits jetzt erfüllen und Ontario, Manitoba sowie Alberta entsprechende Werte bis 2018 anstreben, besteht Handlungsbedarf vor allem im klimapolitisch skeptischen Saskatchewan und in den kleineren Atlantikprovinzen. Den für 2022 vorgesehenen Preis erreicht bisher jedoch keine der Provinzen.

Insgesamt zeigt sich in Nordamerika ein Wechselspiel zwischen nationaler und regionaler Ebene, in dem fortschrittliche Bundesstaaten und Provinzen als Impulsgeber und Experimentierfelder agierten, auf die die nationale Ebene später mit Richtlinien reagierte, um so den inter-regionalen Wettbewerb um die besten Lösungen anzureizen und insbesondere die Nachzügler unter Druck zu setzen.

\section{Fazit}

Der Klimawandel ist eine der größten Herausforderungen unserer Zeit, die selbst nach dem Paris Agreement nur bewältigt werden kann, wenn auf allen politischen Ebenen alle verfügbaren Lösungen unvoreingenommen geprüft und sinnvoll kombiniert werden.

Subnationale Treibhausgas-Emissionshandelssysteme können hier einen wichtigen Beitrag leisten. Sie ermöglichen gerade in Fällen nationalen Klimapolitikversagens die kosteneffiziente Erreichung regionaler Klimaschutzziele und können über eine Verknüpfung sogar zusätzliche Effizienzpotenziale erschließen. Im Sinne einer polyzentrischen, neuföderalen Klimapolitik können subnationale Programme als Versuchslabore dienen, in denen politische Instrumente besonders gut an die regionalen Rahmenbedingungen und Präferenzen angepasst werden können. 
Bisherige Erfahrungen mit solchen Systemen in Kanada und den USA bestätigen diese Vorteile. So ist es nicht nur gelungen, wider den nationalen Trend relativ ambitionierte Programme vor allem in Regionen mit ausgeprägten Umweltpräferenzen in der Bevölkerung zu implementieren. Vielmehr dienten die Erfahrungen aus diesen regionalen Versuchslaboren als Orientierung für nationale Leitlinien zur Bepreisung von Treibhausgasen. Statt eines „race-to-the-bottom“ scheint eine vorbildliche regionale Treibhausgas-Bepreisung per Emissionshandel oder auch per Kohlenstoffsteuer eher ein „race-tothe-top“ zu entfachen. Diese Dynamik könnte auch für andere Länder, in denen nationales Politikversagen bezüglich eines markbasierten Klimaschutzes herrscht - Japan und Australien seien hier nur als Beispiele genannt -, den Weg in eine ökologisch anspruchsvolle, kosteneffiziente und sozial gerechte Klimapolitik weisen.

\section{Literatur}

CARB (2013): California Cap on Greenhouse Gas Emissions and MarketBased Compliance Mechanisms. Sacramento.

Daly, H. (1996): Beyond Growth. Boston, Beacon Press.

EDF (2015): Carbon Market California. San Francisco.

Endres, A. (2011): Environmental Economics. Cambridge, Cambridge University Press.

EPA (2015): Carbon Pollution Emission Guidelines for Existing Stationary Sources, Electric Utility Generating Units, Final Rule. Washington D.C.

GdQ (2014): Québec's Cap-and-Trade System for Greenhouse Gas Emission Allowances. Québec.

GoO (2016): Proposed Climate Change Mitigation and Low-Carbon Society Act 2016. Toronto.

ICAP (2016): Emissions Trading Worldwide. Berlin.

Kemfert, C. (2015): Der Emissionshandel ist klinisch tot. In: Unternehmen \& Gesellschaft 1: 74-77.

Keohane, N. et al. (2015): Towards a club of carbon markets. In: Climatic Change. Im Internet unter: DOI 10.1007/s10584-015-1506-z

Lenz, C. et al. (2014): Die Verknüpfung von Emissionshandelssystemen. Kassel, Kassel University Press.

Morotomi, T. (2014): Climate change policy from the bottom up. In: Niizawa, H./Morotomi, T. (Hrsg.): Governing Low-Carbon Development and the Economy. Tokyo, UN University Press: 117-131.

Oates, W.E. (2004): Environmental Policy and Fiscal Federalism. Cheltenham, Edward Elgar.

OECD (2009): Cities, Climate Change, and Multilevel Governance. Paris.

Ostrom, E. (2009): A Polycentric Approach for Coping with Climate Change. Washington D.C., World Bank Policy Research Working Paper 5095.

Pedersen, T.F./Elgie, S. (2015): A template for the world - British Columbia's carbon tax shift. In: Kreiser, L. et al. (Hrsg.): Environmental taxation and emissions trading in an era of climate change. Cheltenham, Edward Elgar: $3-15$.

Pooley, Eric (2010): The Climate War. New York, Hyperion.

Potomac Economics (2016): Annual Report on the Market for RGGI CO $\mathrm{Al}$ lowances. New York.

Ranson, M./Stavins, R.N. (2015): Linkage of greenhouse gas emissions trading systems: In: Climate Policy. Im Internet unter: DOI 10.1080/14693062.2014.997658

RGGI (2013): Model Rule. New York.

RGGI (2016): $\mathrm{CO}_{2}$ Emissions from Electricity Generation and Imports in the Regional Greenhouse Gas Initiative. New York.

Rudolph, S. et al. (2012): Towards sustainable carbon markets. In: Kreiser, L. et al. (Hrsg.): Carbon Pricing, Growth and the Environment. Cheltenham, Edward Elgar: 167-183.
Rudolph, S. et al. (2014): Regional market-based climate policy in North America In: Kreiser, L. et al. (Hrsg.): Environmental Taxation and Green Fiscal Reform for a Sustainable Future - Theory and Impact. Cheltenham: Edward Elgar: 273-288.

Rudolph, S. et al. (2016): Developing the North American Carbon Market. Kyoto University Graduate School of Economics Discussion Paper No. E-16-009.

Rudolph, S./Lerch, A. (2012): Treibhausgas-Emissionshandel in den USA. In: Zeitschrift für Umweltpolitik \& Umweltrecht 35/4: 421-449.

Rudolph, S./Morotomi, T. (2015): Lokal handeln! In: Zeitschrift für Umweltpolitik und Umweltrecht 38/4: 269-290.

Schmalensee, R./Stavins, R. (2015): Lessons Learned from Three Decades of Experience with Cap-and-Trade. Harvard Kennedy School Faculty Research Working Paper Series RWP15-069.

Trudeau, J. (2016): Justin Trudeau gives provinces until 2018 to adopt carbon price plan. Im Internet unter: http://www.cbc.ca/news/politics/canadatrudeau-climate-change-1.3788825

Under2 (2016): The Memorandum of Understanding (MOU) on Subnational global Climate Leadership. Im Internet unter: http://under2mou.org/ the-mou/

WBGU (2010): Klimapolitik nach Kopenhagen. Politikpapier Nr. 6. Berlin.

WCI (2008): Design Recommendations for the WCI Regional Cap-and-Trade Program,. Sacramento.

\section{AUTOREN + KONTAKT}

Dr. Sven Rudolph und Toru Morotomi sind Professoren an der Kyoto University, Takeshi Kawakatsu ist Professor an der Kyoto Prefectural University, Japan.

Dr. Achim Lerch ist Professor für allgemeine Volkswirtschaftslehre an der FOM Hochschule für Ökonomie und Management, Kassel.

Gemeinsam arbeiten sie derzeit an Fragen der subnationalen marktbasierten Klimapolitik.

Assoc. Prof. Dr. Sven Rudolph, Kyoto University, Graduate School of Economics, Yoshida-Honmachi, Sakyo-ku, Kyoto-shi 606-8501, Japan. Tel.: +81 75753 344, E-Mail: rudolph@econ.kyoto-u.ac.jp
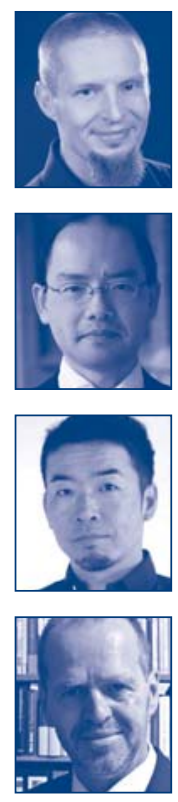